Reprod. Nutr. Dévelop., 1988, 28 Suppl. n¹, 93-94

\title{
Etude en fermenteur semi-continu de l'influence de l'addition de sulfate sur la dégradation des constituants pariétaux d'une paille non traitée ou traitée à la soude
}

Jocelyne STEVANI, Ph. BEAUMATIN, Christiane DUMAY, Geneviève HANNEQUART, Michelle DURAND

Station de Recherches de Nutrition, I.N.R.A.

78350 Jouy-en-Josas, France.

Summary. The effect of sulphur (S) on the cell-wall degradation of wheat straw either untreated or alkali-treated by rumen microbes has been studied using the Rusitec technique. $\mathrm{S}$ addition had no effect on untreated straw but increased the degradation of all neutral sugars, especially glucose, and the cellulase specific activities when straw was alkali-treated.

Le soufre (S) est nécessaire aux microorganismes du rumen pour la biosynthèse des acides aminés soufrés à partir d'azote non protéique. La supplémentation en $S$ des fourrages pauvres pourrait également améliorer la cellulolyse (Durand et Komisarczuk, 1988). Nous avons cherché à approfondir ce dernier aspect en étudiant l'influence d'une supplémentation en sulfate sur la dégradation par les microorganismes du rumen des constituants pariétaux d'une paille avant et après traitement alcalin.

Matériel et méthodes. Deux séries d'études sont menées en fermenteur semi-continu (Rusitec), selon la méthodologie générale rapportée par Durand et al. (1988). La paille de blé hachée contenant $1,3 \mathrm{~g} \mathrm{~S} / \mathrm{kg}$ de matière sèche (MS) $(\mathrm{S} / \mathrm{N}=0,4)$, a été introduite dans des sachets nylon (vide de maille $150 \mu \mathrm{m})$ à raison de $16 \mathrm{~g}$ de $\mathrm{MS} / \mathrm{jour} / \mathrm{sac}$. Selon l'essai, cette paille a été utilisée soit non traitée, soit après traitement à la soude $0,4 \mathrm{M}(2,5 \mathrm{l} / \mathrm{kg} \mathrm{MS})$. Dans chacun des essais, deux fermenteurs témoins reçoivent un mélange tampon, pauvre en $S$ $(0,6 \mathrm{mg} \mathrm{S} / \mathrm{l})$ et deux fermenteurs supplémentés, un mélange tampon enrichi en $\mathrm{Na}_{2} \mathrm{SO}_{4}(16,4 \mathrm{mg} \mathrm{S} / \mathrm{l})$. Les tampons additionnés d'urée (apport calculé sur la base de $40 \mathrm{~g} \mathrm{~N} / \mathrm{kg}$ de matière organique fermentescible (MOF), soit 140 et $190 \mathrm{mg} \mathrm{N} / \mathrm{l}$, respectivement pour la paille brute et la paille traitée) sont perfusés en continu ( $1 \mathrm{l} /$ jour/fermenteur). Après 7 jours d'adaptation, les résidus contenus dans les sachets sont récoltés pendant 4 jours et sont cumulés sur deux jours consécutifs pour un même fermenteur. La teneur des résidus en composés pariétaux est déterminée par la méthode classique aux détergents (Goering et Van Soest, 1970) et après hydrolyse acide par chromatographie en phase gazeuse des oses neutres dérivés sous forme d'acétates d'alditol selon une méthode adaptée de Blakeney et al. (1983). L'activité des cellulases de la phase liquide associée aux particules (phase intermédiaire) prélevée deux fois pour chaque fermenteur a été évaluée dans l'essai sur la paille traitée par la méthode de Forsberg et al. (1981). 
Résultats et discussion. Pour la paille il est possible d'assimiler la fraction cellulose à la totalité du glucose, et la fraction hémicellulosique à la somme xylose + arabinose. Cependant, la dégradabilité des hémicelluloses de la paille traitée supplémentée ou non (tabl. 1), déterminée par la méthode aux détergents est plus faible que celle obtenue par le dosage des oses neutres. Ceci peut être attribué à la sous-estimation des hémicelluloses rendues plus labiles par le traitement avec la méthode aux détergents.

TABL. 1. - Effet de l'addition de $S$ sur la dégradation des constituants pariétaux.

\begin{tabular}{|c|c|c|c|c|c|c|}
\hline & \multicolumn{2}{|c|}{ Teneurs (\%) } & \multicolumn{4}{|c|}{ Dégradabilité ( $\%$ de l'introduit) $(n=4)$} \\
\hline & \multirow{2}{*}{$\begin{array}{l}\text { Paille } \\
\text { brute }\end{array}$} & \multirow{2}{*}{$\begin{array}{l}\text { Paille } \\
\text { traitée }\end{array}$} & \multicolumn{2}{|c|}{ Paille brute } & \multicolumn{2}{|c|}{ Pailie traitée } \\
\hline & & & $-\mathrm{s}$ & $+s$ & $-\mathrm{s}$ & $+\mathrm{S}$ \\
\hline Cellulose (ADF-lignine) & $42^{\mathrm{a}}$ & $42^{\mathrm{a}}$ & $33,0^{\mathrm{b}}$ & $34,8^{\mathrm{b}}$ & $42,1^{\mathrm{c}}$ & $59,2^{d}$ \\
\hline Hémicelluloses (NDF-ADF) & $33^{\mathrm{a}}$ & $23^{\mathrm{b}}$ & $32,7^{\mathrm{c}}$ & $34,6^{\mathrm{c}}$ & $38,0^{d}$ & $53,0^{\circ}$ \\
\hline Glucose & $41^{\mathrm{a}}$ & $39^{\mathrm{a}}$ & $36,9^{b}$ & $39,6^{\mathrm{b}}$ & $37,8^{\mathrm{c}}$ & $56,7^{d}$ \\
\hline Xylose & $23^{\mathrm{a}}$ & $25^{\mathrm{a}}$ & $37,4^{\mathrm{b}}$ & $42,5^{\mathrm{b}}$ & $49,9^{\mathrm{c}}$ & $63,2^{\mathrm{d}}$ \\
\hline Arabinose & $6,6^{\mathrm{a}}$ & $6,0^{a}$ & $74,5^{b}$ & $73,6^{\mathrm{b}}$ & $44,3^{\mathrm{c}}$ & $58,4^{d}$ \\
\hline
\end{tabular}

$\mathrm{a} \neq \mathrm{b} \neq \mathrm{c}: \mathrm{P}<0,01$.

L'addition de $S$ qui n'améliore la dégradabilité d'aucun constituant de la paille brute de façon significative a, par contre, un effet largement bénéfique sur la dégradation des diverses fractions pariétales de la paille traitée. Celle-ci est nécessaire à la pleine expression de l'effet de traitement à la soude : ce traitement améliore la cellulolyse de $70 \%$ dans les fermenteurs supplémentés et seulement de $28 \%$ dans les fermenteurs témoins. L'accroissement de la dégradation est identique pour les hémicelluloses et la cellulose mesurées par la méthode aux détergents $(+40 \%)$; par contre, le dosage des oses neutres met en évidence un effet beaucoup plus marqué sur le glucose $(+50 \%)$ que sur la fraction xylose + arabinose $(+28 \%)$. Ces observations corroborent les résultats du dosage des cellulases de la phase intermédiaire où la concentration en enzymes est deux fois plus importante pour les fermenteurs supplémentés que pour les fermenteurs témoins (respectivement 10,8 et $5,4 \mu \mathrm{g}$ de glucose libéré $/ \mathrm{min} / \mathrm{ml}$ ). Les activités spécifiques (cellulases/protéines) montrent un écart moins important mais encore très significatif (soit 22,9 contre 16,9 $\mathrm{\mu g}$ de glucose libéré $/ \mathrm{min} / \mathrm{mg}$ de protéines). Le soufre a donc une action directe sur l'activité des cellulases et sur la quantité globale d'enzymes produite par la biomasse microbienne.

En conclusion, la supplémentation en S est nécessaire à l'optimisation de la dégradation de la paille traitée alors que l'apport intrinsèque de $S$ du substrat suffit à celle de la dégradation de la paille brute. Ces résultats mettent en évidence l'accroissement des besoins en $\mathrm{S}$ disponible parallèlement à l'augmentation de la MOF et implique la nécessité d'exprimer ces besoins par rapport à la MOF et non par rapport à la $\mathrm{MS}$ ou au rapport $\mathrm{S}$ total $/ \mathrm{N}$.

Blakeney A. B., Harris P. J., Henry R. J., Stone B. A., 1983. Carbohyd. Res., 113, 291-299. Durand M., Komisarczuk S., 1988 . J. Nutr., 118, 249-260.

Durand M., Dumay C., Beaumatin P., Morel M. T., 1988. Anim. Feed Sci., Technol., 21, 197-204.

Forsberg C. W., Beveridge T. J., Hellstrom A., 1981. App/. Environ. Microbiol., 42, 886-896.

Goering H. K., Van Soest P. J., 1970 . Forage fiber analyses. Agriculture Handbook $n^{\circ} 379$, Agric. Res. Serv. U.D. Department of Agriculture. 